3. Lauer SA, Grantz KH, Bi Q, et al. The incubation period of coronavirus disease 2019 (COVID-19) from publicly reported confirmed cases: estimation and application. Ann Intern Med 2020;172:577-582.

4. Backer JA, Klinkenberg D, Wallinga J. Incubation period of 2019 novel coronavirus (2019-nCoV) infections among travellers from Wuhan, China, 20-28 January 2020. Euro Surveill 2020;25(5):2000062.

5. Leung C. The difference in the incubation period of 2019 novel coronavirus (SARS-CoV-2) infection between travelers to Hubei and nontravelers: the need for a longer quarantine period. Infect Control Hosp Epidemiol 2020; 41:594-596.

6. Nie X, Fan L, Mu G, et al. Epidemiological characteristics and incubation period of 7,015 confirmed cases with coronavirus disease 2019 outside Hubei Province in China. J Infect Dis 2020;222:26-33.
7. Linton NM, Kobayashi T, Yang Y, et al. Incubation period and other epidemiological characteristics of 2019 novel coronavirus infections with right truncation: a statistical analysis of publicly available case data. J Clin Med 2020;9(2): 538.

8. Coronavirus disease 2019 (COVID-19) quarantine and isolation. Centers for Disease Control and Prevention website. https://www.cdc.gov/coronavirus/ 2019-ncov/if-you-are-sick/quarantine-isolation.html. Accessed June 6, 2020.

9. Kucirka LM, Lauer SA, Laeyendecker O, Boon D, Lessler J. Variation in false-negative rate of reverse transcriptase polymerase chain reaction-based SARS-CoV-2 tests by time since exposure. Ann Intern Med 2020. doi: 10. 7326/M20-1495.

10. Wang Y, Wang Q, Wang K, Song C, Guo Z, Hu W. A case of COVID-19 with ultra-long incubation period. Infect Control Hosp Epidemiol 2020. doi: 10.1017/ice.2020.221.

\title{
Patients' anxiety, fear, and panic related to coronavirus disease 2019 (COVID-19) and confidence in hospital infection control policy in outpatient departments: A survey from four Thai hospitals
}

\author{
Anucha Apisarnthanarak MD ${ }^{1}$, Chanida Siripraparat MD, MPH², Piyaporn Apisarnthanarak MD³ , Michael Ullman $\mathrm{PhD}^{4}$, \\ Pavarat Saengaram MD ${ }^{5}$, Narakorn Leeprechanon $\mathrm{MD}^{6}$ and David J. Weber $\mathrm{MD}^{7}$ \\ ${ }^{1}$ Division of Infectious Diseases, Faculty of Medicine, Thammasat University, Prathum Thani, Thailand, ${ }^{2}$ Manorom Hospital, Bangkok, Thailand, ${ }^{3}$ Division of \\ Diagnostic Radiology, Department of Radiology, Faculty of Medicine Siriraj Hospital, Mahidol University, Bangkok, Thailand, ${ }^{4}$ Research and Consulting Service, \\ Michael Ullmann Consulting, Baltimore, Maryland, United States, ${ }^{5}$ Bumrungrad Hospital, Bangkok, Thailand, ${ }^{6}$ Department of Ophthalmology, Rutnin Hospital, \\ Bangkok, Thailand and ${ }^{7}$ Division of Infectious Diseases, University of North Carolina, Chapel Hill, North Carolina, United States
}

To the Editor-The emergence of the coronavirus disease 2019 (COVID-19) pandemic has disrupted day-to-day patient life with limitations to social practices (eg, physical distancing, mask wearing, and frequent hand hygiene). ${ }^{1}$ These limitations, together with widespread anxiety and stress, have generated a mental health crisis among patients. ${ }^{2}$ Anxiety, fear and panic related to COVID-19 may result in strong emotions and reactions. ${ }^{1-3}$ Therefore, we conducted a survey to evaluate COVID-19associated patient emotions and confidence in hospital infection prevention (IP) and IP behaviors in outpatient departments.

This survey was performed at 2 university hospitals and 2 private hospitals from May 1 to May 30, 2020. To represent multiple patient populations, patients visiting 3 outpatient departments (general medicine, ophthalmology, and radiology) were invited to participate in the study and were interviewed using a standardized data collection tool. The first 50 patients who filled out the survey in each hospital were included in the data analysis. The data collected included patient demographics, perception of risks to contract COVID-19, confidence in policy and preparedness plan for COVID-19, sources of knowledge, and emotions evoked by COVID-19, and IP practices (eg, hand hygiene, wearing a mask, and physical distancing). Respondents rated their confidence level on knowledge and hospital preparedness plan on a scale

Author for correspondence: Anucha Apisarnthanarak, E-mail: anapisarn@yahoo.com Cite this article: Apisarnthanarak A, et al. (2021). Patients' anxiety, fear, and panic related to coronavirus disease 2019 (COVID-19) and confidence in hospital infection control policy in outpatient departments: A survey from four Thai hospitals. Infection Control \& Hospital Epidemiology, 42: 1288-1290, https://doi.org/10.1017/ice.2020.1240 from 1 to 5 (1, "no confidence" to 5 , "very confident") as well as changing in IP behaviors on a scale from 1 to 5 (1, "never use" to 5 , "always use"). IP behavior changes (eg, hand hygiene, wearing a mask, and physical distancing) were defined as a rating of 4 (almost always) or 5 (always). We used the Generalized Anxiety Disorder 7-item (GAD-7) scale to categorize anxiety, self-rated fear, and panic on a scale from 1 to 10 (1, "no fear/panic" to 10 , "extreme fear/panic"). The categorization of the GAD-7 score followed the original scale (ie, $0-4$, minimal anxiety; 5-9, mild anxiety; $10-14$, moderate anxiety; and $>14$, severe anxiety), ${ }^{4}$ and self-reported fear $>6$ was categorized as fear of COVID- 19 .

All analyses were performed using SPSS version 19 software (IBM, Armonk, NY). The $\chi^{2}$ or Fisher exact test was used to compare categorical variables. The Mann-Whitney $U$ test was used for continuous data. All $P$ value were 2 -tailed, and $P<.05$ was considered statistically significant. Multivariate analysis was used to evaluate factors associated with emotions and impact of emotions on IP practices.

In total, 200 patients participated in this survey ( $\mathrm{n}=50$ patients per hospital). The median age of respondents was 45 years (range, $15-92)$, and 138 of 200 participants (70\%) were women. Some patients reported having had contact with COVID-19 patients or a patient under investigation (19 of 200, 9.6\%). Anxiety, fear, and panic related to COVID-19 were reported by 181 of 200 (90\%), 89 of $200(45 \%)$, and 82 of $200(41 \%)$, respectively. Feelings of discrimination and stigma against COVID-19 patients were reported by 113 of $200(57 \%)$ and 107 of $200(54 \%)$, respectively. Social media ( 164 of $200,83 \%$ ) was the most common source

(c) The Author(s), 2020. Published by Cambridge University Press on behalf of The Society for Healthcare Epidemiology of America. This is an Open Access article, distributed under the terms of the Creative Commons Attribution licence (http://creativecommons.org/licenses/by/4.0/), which permits unrestricted re-use, distribution, and reproduction in any medium, provided the original work is properly cited. 
Table 1. Patients Characteristics, Emotions, Confidence in Hospital Infection Prevention Practices at Outpatient Departments During the COVID-19 Pandemic

\begin{tabular}{|c|c|}
\hline Variables & $\begin{array}{l}\text { Total }(\mathrm{N}=200) \\
\text { No. }(\%)\end{array}$ \\
\hline Age, median y (range) & $45(15-92)$ \\
\hline Sex, female & $138(69)$ \\
\hline \multicolumn{2}{|l|}{ Occupation } \\
\hline Employee & $41(20.7)$ \\
\hline Business man & $35(17.3)$ \\
\hline Government worker & $22(11)$ \\
\hline Others $^{\mathrm{a}}$ & $102(51)$ \\
\hline \multicolumn{2}{|l|}{ Type of mask } \\
\hline Cloth mask & $113(57)$ \\
\hline Surgical mask & $71(36)$ \\
\hline N95 mask & $11(5.6)$ \\
\hline Others ${ }^{b}$ & $5(2.5)$ \\
\hline $\begin{array}{l}\text { Contact with COVID-19 patients or patient under } \\
\text { investigation }\end{array}$ & $19(19.6)$ \\
\hline Fear for contracting COVID-19 & $89(45)$ \\
\hline Panic for being contracting COVID-19 & $82(41.4)$ \\
\hline Confidence in hospital preparedness policy & $175(88)$ \\
\hline Confidence in hospital hand hygiene policy & $196(99)$ \\
\hline $\begin{array}{l}\text { Confidence in wearing mask policy at outpatient } \\
\text { department }\end{array}$ & $187(94)$ \\
\hline $\begin{array}{l}\text { Confidence in social distancing policy at } \\
\text { outpatient department }\end{array}$ & $163(82)$ \\
\hline Confidence in COVID-19 knowledge & $150(76)$ \\
\hline \multicolumn{2}{|l|}{ Source of COVID-19 information } \\
\hline \multicolumn{2}{|l|}{ Social media } \\
\hline Line app & $164(83.5)$ \\
\hline Facebook & $135(67)$ \\
\hline Instragram & $154(77)$ \\
\hline Government news & $171(86)$ \\
\hline Television news & $174(87)$ \\
\hline Feeling of discrimination & $113(57)$ \\
\hline Feeling of stigmatization & $107(54)$ \\
\hline \multicolumn{2}{|l|}{ GAD-7 Score } \\
\hline Mild anxiety & $155(78)$ \\
\hline Moderate anxiety & $15(7.6)$ \\
\hline Severe anxiety & $11(5.6)$ \\
\hline \multicolumn{2}{|l|}{ Changing in infection control behavior } \\
\hline Hand washing & $140(70)$ \\
\hline Wearing mask & $124(62)$ \\
\hline $\begin{array}{l}\text { Social distancing at workplace and outpatient } \\
\text { department }\end{array}$ & $159(79)$ \\
\hline
\end{tabular}

Note. PPE, personal protective equipment; HCP, healthcare personnel; GAD-7, Generalized Anxiety Disorder 7-items.

aStudents, healthcare personnel, housewife, unemployment, self-employed.

${ }^{\mathrm{b}}$ Self-made mask. of COVID-19 information among patients (Table 1). There were no differences in patients' characteristics between private and university hospitals.

Most patients ( 175 of $200,88 \%$ ) expressed confidence in the overall hospital IP policy. Patient confidence in policies was as follows: hand hygiene (196 of 200, 99\%), physical distancing (163 of 200, $82 \%$ ), and mask wearing in the outpatient department (187 of $200,94 \%)$. Only 159 of $200(80 \%)$ reported that the hospital had adequate PPE for patients. Most patients reported changing behavior with more frequent hand hygiene (140 of $200,70 \%)$, wearing mask at workplace or hospital (124 of 200,62\%), complying with physical distancing at workplace or hospital (159 of 200,79\%), and 150 of 200 (76\%) expressed confidence in their knowledge of severe acute respiratory coronavirus virus 2 (SARS-CoV-2) transmission (Table 1).

By multivariate analysis, no factor was associated with anxiety, fear, and panic. However, patients who reported anxiety and panic were more likely to wear a mask at the workplace or hospital (adjusted odds ratio [aOR], 5.4; 95\% CI, 1.7-45.5), and patients who reported fear were more likely to wear mask at the workplace or hospital (aOR, $6.4 ; 95 \% \mathrm{CI}, 1.8-52.6)$ and to wash hands more frequently (aOR, 5.7; $95 \%$ CI, 1.7-51.5). Notably, patients who reported having good information regarding SARS-CoV-2 transmission were more likely to comply with physical distancing policy at the workplace or hospital (aOR, 4.2; 95\% CI, 1.2-15.4), to wash hands more frequently (aOR, $5.9 ; 95 \% \mathrm{CI}, 1.5-22)$, and to wear a mask at the workplace or hospital (aOR, 4.9; 95\% CI, 1.3-18.9).

Our findings suggest that most patients were overwhelmed with anxiety, fear, and panic during the COVID-19 epidemic, despite a high level of confidence in hospital IP practices. Although these emotions as well as information regarding SARS-CoV-2 transmission led to changing their behavior (eg, hand hygiene, wearing a mask and physical distancing), we found that a significant proportion of patients were feeling discrimination and stigma toward COVID19 patients. Thus, education on SARS-CoV-2 transmission should be provided in a way that does not trigger feelings of fear, anxiety, panic, discrimination, and stigmatization because these feelings may lead to violence in the community toward COVID-19 patients. $^{5}$

Most patients had confidence in the hospital preparedness policy in outpatient departments; however, the level of changes in IP practices was still less than ideal. Therefore, additional strategies to enhance the level of IP practices in outpatient department are needed. Furthermore, several mask types are used in these patient populations (eg, surgical masks and N95 respirators). Education to emphasize the use of nonmedical masks among patients during outpatient visits is necessary.

Despite the limitation of self-reported survey and the sample size in this study, our study supports the need for hospitals to continuously provide information regarding SARS-CoV-2 transmission and an adequate supply of masks as well as emphasizing education on IP practices in outpatient departments. Additional studies on the impact of SARS-CoV-2 transmission knowledge on appropriate IP behaviors as well as perception of discrimination and stigmatization against COVID-19 patients should be conducted.

\section{Acknowledgments.}

Financial support. No financial support was provided relevant to this article.

Conflicts of interest. All authors report no conflicts of interest relevant to this article. 


\section{References}

1. Lima CKT, Carvalho PMM, Lima IAAS, et al. The emotional impact of coronavirus 2019-nCOV (new coronavirus disease). Phychiatry Res 2020;287: 112915.

2. Li W, Yang Y, Liu $\mathrm{ZH}$, et al. Progression of mental health service during COVID-19 outbreak in China. Int J Biol Sci 2020;16:1732-1738.
3. Lu W, Wang H, Lin Y, Li L. Psychological status of medical workforce during the COVID-19 pandemic: a cross-sectional study. Psychiatry Res 2020; 288:112936.

4. Spitzer RL, Kroenke K, Williams JB, Lowe B. A brief measure for accessing generalized anxiety disorder: the GAD-7. Arch Intern Med 2006;166: 1092-1097.

5. Bruns DP, Kraguljac NV, Bruns TR. COVID-19: facts, cultural consideration and risk of stigmatization. J Transcult Nurs 2020;31:326-332.

\title{
May we learn a useful lesson from prevention rules against severe acute respiratory coronavirus virus 2 (SARS-CoV-2)?
}

\author{
Silvia Corbellini MD, Maria Antonia De Francesco PhD and Arnaldo Caruso PhD \\ Department of Molecular and Translational Medicine, Institute of Microbiology, University of Brescia-ASST Spedali Civili di Brescia, Brescia, Italy
}

To the Editor-The emergence and diffusion of oxacillin-resistant Staphylococcus aureus (MRSA) constitutes an important problem for public health. Data from European countries reported a trend with an increasing MRSA prevalence from the north to the south of the continent: $<5 \%$ of MRSA has been isolated from invasive infections in north of Europe compared with $25 \%-50 \%$ in the south of Europe. ${ }^{1}$

This gram-positive bacterium is generally found as part of commensal flora in the nasal mucosa in $20 \%-40 \%$ of the population and just these people, who are asymptomatic carriers, have an increased risk to acquire a subsequent infection in addition to representing an important source of person-to-person transmission. In particular, hospital and healthcare settings represent a favorable environment that predispose to infection because of a high antibiotic selection pressure, the use of invasive procedures, and the presence of critically ill patients. For these reasons, MRSA is now endemic in many hospitals worldwide, and infection control measures are needed to prevent its transmission, especially considering the risk of development of glycopeptide-resistant $S$. aureus strains.

Hospital control of endemic MRSA has been based on standard precautions such as isolation/cohorting, hand hygiene, patient decolonization, and appropriate use of antibiotic (antibiotic stewardship). Intensive care units of Spedali Civili's Hospital of Brescia has implemented active surveillance cultures to identify patients who acquire MRSA during hospitalization. This surveillance involves nasal swabs for the screening of patients at the time of hospital admission to identify asymptomatic carriers, followed by periodic screening every 3 days.

One of these intensive care units became a coronavirus disease 2019 (COVID-19) ward during the pandemic, and we analyzed whether the higher compliance to the use of personal protective equipment (PPE, eg, gloves, coveralls, face mask and boots) by all the hospital staff had an impact on the prevalence of MRSA acquisition during patients hospitalization.

Author for correspondence: Arnaldo Caruso, E-mail: arnaldo.caruso@unibs.it

Cite this article: Corbellini S, De Francesco MA, and Caruso A. (2021). May we learn a useful lesson from prevention rules against severe acute respiratory coronavirus virus 2 (SARS-CoV-2)? Infection Control \& Hospital Epidemiology, 42: 1290-1291, https:// doi.org/10.1017/ice.2020.1228
Table 1. Trend of MRSA Detection During the Study Period

\begin{tabular}{|c|c|c|c|}
\hline & 2019 & 2020 & \\
\hline Year & No. of Positive/Total (\%) & No. of Positive/Total (\%) & $P$ Value \\
\hline January & $9 / 103(8)$ & 4/134 (3) & .08 \\
\hline February & $10 / 102(9)$ & $2 / 126(2)$ & .01 \\
\hline March & $18 / 135(12)$ & $5 / 274(2)$ & .0001 \\
\hline April & $13 / 108(11)$ & $7 / 269(3)$ & .01 \\
\hline May & $25 / 97(20)$ & $4 / 193(2)$ & .0001 \\
\hline June & $21 / 83(20)$ & $2 / 178(1)$ & .0001 \\
\hline July & $17 / 97(15)$ & $0 / 183(0)$ & .0001 \\
\hline August & $21 / 107(16)$ & $8 / 153(5)$ & .01 \\
\hline Total & $134 / 832(14)$ & $32 / 1510(2)$ & .0001 \\
\hline
\end{tabular}

It is well known that healthcare workers can transmit infections such as tuberculosis, varicella and influenza by the airborne route, ${ }^{2}$ but it less well known that airborne and other ways of transmission may occur with some bacterial pathogens. In particular, the use of face masks prevents pathogen transmission from the wearer to other people and reduces hand-to-face contact and facial contact with droplets. ${ }^{3}$

In our analysis, we compared the MRSA detection after 48 hours following hospital admission during January-August 2020 versus January-August 2019. As shown in Table 1, we observed a statistically significant reduction in the prevalence of nosocomially acquired MRSA ( $2 \%$ vs $14 \%$; $P<.0001)$. This decrease was always statistically significant for all the months analyzed except January, when the implementation of PPE in the absence of COVID-19 was not present (Table 1). This finding is not surprising, since a previous study showed that a healthcare worker, who did not wear a mask and who was a nasal carrier of MRSA, induced a 40 -fold increase in MRSA dispersion. ${ }^{4}$

Although our preliminary data need to be confirmed by larger studies, our observation suggests implementation of PPE as a strong preventive strategy to control hospital-acquired MRSA infection. 